\title{
TU/e EmonOWEN

\section{Adaptive coordination of sequential droop control for PV inverters to mitigate voltage rise in PV-Rich LV distribution networks}

Citation for published version (APA):

Mai, T., Haque, A. N. M. M., Vergara Barrios, P., Nguyen, P. H., \& Pemen, A. J. M. (2021). Adaptive coordination of sequential droop control for PV inverters to mitigate voltage rise in PV-Rich LV distribution networks. Electric Power Systems Research, 192, [106931]. https://doi.org/10.1016/j.epsr.2020.106931

\section{Document license:}

TAVERNE

DOI:

10.1016/j.epsr.2020.106931

Document status and date:

Published: 01/03/2021

\section{Document Version:}

Publisher's PDF, also known as Version of Record (includes final page, issue and volume numbers)

\section{Please check the document version of this publication:}

- A submitted manuscript is the version of the article upon submission and before peer-review. There can be important differences between the submitted version and the official published version of record. People interested in the research are advised to contact the author for the final version of the publication, or visit the DOI to the publisher's website.

- The final author version and the galley proof are versions of the publication after peer review.

- The final published version features the final layout of the paper including the volume, issue and page numbers.

Link to publication

\footnotetext{
General rights

- You may freely distribute the URL identifying the publication in the public portal. follow below link for the End User Agreement:

www.tue.nl/taverne

\section{Take down policy}

If you believe that this document breaches copyright please contact us at:

openaccess@tue.nl

providing details and we will investigate your claim.
}

Copyright and moral rights for the publications made accessible in the public portal are retained by the authors and/or other copyright owners and it is a condition of accessing publications that users recognise and abide by the legal requirements associated with these rights.

- Users may download and print one copy of any publication from the public portal for the purpose of private study or research.

- You may not further distribute the material or use it for any profit-making activity or commercial gain

If the publication is distributed under the terms of Article $25 \mathrm{fa}$ of the Dutch Copyright Act, indicated by the "Taverne" license above, please 


\title{
Adaptive coordination of sequential droop control for PV inverters to mitigate voltage rise in PV-Rich LV distribution networks
}

\author{
Tam T. Mai ", Abu Niyam M.M. Haque, Pedro P. Vergara, Phuong H. Nguyen, Guus Pemen \\ Electrical Energy Systems (EES) Group, Department of Electrical Engineering, Eindhoven University of Technology, Den Dolech 2, 5612AZ Eindhoven, The Netherlands
}

\section{A R T I C L E I N F O}

\section{Keywords:}

Active power curtailment

Reactive power absorption

Sequential droop control

Consensus algorithm

Distributed control

Network decomposition

\begin{abstract}
A B S T R A C T
This paper introduces an adaptive sequential droop control strategy for PV inverters to mitigate voltage rise problems in PV-rich LV distribution networks. To facilitate the effective coordination of sequential $(Q-V$ and $P-V$ ) droop control of PV inverters, multiple control areas with the strong coupling nature of PV systems are identified based on the $\varepsilon$-decomposition technique. The droop control parameters are tuned and adapted, based on a consensus among PV inverters within each control area. This proposed control strategy inherits the autonomous feature of the droop control for coping with voltage rise issues while being able to avoid curtailing a significant amount of PV production. To evaluate the effectiveness of the proposed control strategy, simulations using MATLAB/Simulink are performed on a real European LV distribution network, considering a PV penetration level of about $150 \%$. The obtained results highlight that the proposed control strategy successfully mitigates voltage rise problems while significantly reducing the amount of curtailed PV generation by approximately $35.6 \%$ and $76.2 \%$ when compared with the static sequential droop control and the static $Q-V$ droop control and adaptive $P-V$ droop control, respectively. Simultaneously, the effective contribution among all the PV systems towards voltage rise mitigation is obtained.
\end{abstract}

\section{Introduction}

Recently, the integration of residential-scale PV systems is accelerating in low-voltage (LV) distribution networks, which poses technical challenges regarding power quality and reliability [1]. One of the foremost issues is the voltage rise, which becomes a major limiting factor for further PV deployment [2]. Lack of proper solutions to this problem results in on-off switching of PV units activated by the internal protection [3]. Subsequently, this leads to loss of energy yields of PV systems as well as causes damages to the electrical appliances.

To address voltage rise problems, a number of solutions can be found in the literature, including network reinforcement, capacitor bank installations, transformer tap changer adjustment, electrical energy storage-based methods, and PV inverter control [4,5]. Among these, controlling PV inverters to regulate its output active and/or reactive powers emerges as a promising solution [6], especially considering that they can be implemented locally, embedded in the inverter's control system, while simultaneously complementing the already existing voltage control infrastructure. Various control methods based on reactive power absorption (RPA) have been proposed [7-10]. These methods apply control rules based on the network state, e.g., voltage magnitude or power quality indices, to regulate the amount of absorbed reactive power by the inverters. The voltage magnitude at the point of connection (POC) of the inverter, subsequently, is reduced. The RPA scheme can be implemented using $\cos \phi(P), \cos \phi(P V)$, or $Q-V$ droop control, of which $Q-V$ droop control is widely used [9]. Nevertheless, RPA by itself is unable to sufficiently solve voltage rise problems due to the high $R / X$ ratios in $L V$ distribution networks, and the limited reactive power capacity of the PV inverters [4]. Alternatively, active power curtailment (APC), which decreases output active power of PV inverters to reduce the voltage level at the POC, has shown to be more effective $[11,12]$. The APC scheme can be based on $P-V$ droop control or voltage/active power sensitivity [13]. Among these, the $P-V$ droop control is most widely utilized [14]. However, this scheme directly leads to losses of PV owners' revenue due to generation reductions. A combination of RPA and APC has been reported in [15-17] to be more promising for voltage rise mitigation. This type of combined method helps to mitigate voltage rise issues with a decreased amount of curtailed

\footnotetext{
* Corresponding author.

E-mail addresses: t.t.mai@tue.nl (T.T. Mai), a.n.m.m.haque@tue.nl (A.N.M.M. Haque), p.p.vergara.barrios@tue.nl (P.P. Vergara), p.nguyen.hong@tue.nl (P.H. Nguyen), a.j.m.pemen@tue.nl (G. Pemen).
} 
active power, as shown in [18]. However, a major disadvantage of this approach is the unfair contribution of active power curtailment among PV units to voltage rise prevention. This brings PV owners to a disadvantageous position as they have fewer opportunities to export the surplus PV generation. Thus, they might cease to deploy the voltage rise prevention or even protest against it [19]. Hence, from the customer perspective, the unfair curtailment should be effectively managed.

One of the solutions to the effective contribution of PV systems towards mitigating voltage rise problems is the fairness of active power curtailment as proposed in [20-24]. The work in [20] adopted the sensitivity matrix to fairly curtail active power. However, reactive power control of PV inverters was not considered while the method fully relied on a central controller. The method in [21] combined RPA and APC scheme for PV inverters, where the coordinated control algorithm is implemented by a central controller. Such centralized methods heavily depend upon extensive high-bandwidth communication networks along with a large number of interconnected devices making the system vulnerable to a single point-of-failure and costly to be deployed [25]. In contrast, distributed control uses a sparse communication network with a significantly low bandwidth requirement, which enables this type of control architecture to be less costly and less complex. Considering the widespread integration of residential-scale PV units, distributed control is potentially practical to replace centralized control. The method in [22] employed a distributed control scheme to obtain fair generation curtailment of PV systems. However, this method excludes the RPA scheme for PV inverter control. A common feature of these three methods is that the coordinated control algorithm provides the set points of active power values for PV inverters. Meanwhile, the interaction between the droop control and the coordinated control has not been discussed. In [23] an adaptive droop-based approach for PV systems is presented to tackle voltage rise by firstly absorbing reactive power and then fairly curtailing active power. The necessity to know the droop control parameters of other PV systems and a complex signaling pattern to recognize the voltage violation conditions are the main drawbacks of this approach. The study in [24] proposes a static $Q-V$ droop control and an adaptive $P-V$ droop control scheme using consensus-based distributed coordination to prevent voltage rise. For this study, the threshold value for $Q-V$ droop control remains constant over simulation time, while the threshold value for $P-V$ droop control scheme is periodically updated. This method, furthermore, considers the fair curtailment at a feeder level; resulting in a higher total amount of curtailed power when compared with the droop-based local control scheme. Since the impact of PV generation on voltage regulation varies depending on their location [26], grouping PV inverters and coordinating them would be more promising to solve voltage rise problems. The groups of PV inverters can be identified through a network decomposition technique.

Based on the above-presented discussion, this paper introduces an adaptive sequential droop control (ASDC) strategy for PV inverters in a combination with the $\varepsilon$-decomposition technique to alleviate voltage rise problems in PV-rich LV distribution networks. The proposed control strategy is essentially an improvement of our previous work [24] integrating the $\varepsilon$-decomposition technique and the adaptive sequential droop control, in which both RPA and APC schemes are periodically updated, to permit the application in different control areas. While the centralized control scheme can be used to realize the optimization approach for minimizing PV power curtailment, this paper introduces the distributed control scheme based on the consensus algorithm to realize adaptive sequential droop control strategy for coordinating PV inverters. This paper aims to obtain a reduced amount of required PV power reduction compared to the cases with the conventional, i.e, uncoordinated, droop control schemes and the adaptive active power droop control method for adequately solving the voltage rise problems, instead of obtaining an optimal minimum power curtailment. Among all the features discussed, the main contributions of this paper can be summarized as follows:
- A distributed coordination control scheme is proposed that adapts both the $Q-V$ and $P-V$ droop control parameters of the PV inverters to reduce the amount of curtailed generation and guarantee the effective contribution of all PV systems, while alleviating voltage rise problems. Voltage rise mitigation is first attempted through RPA scheme. APC scheme is triggered when the voltage levels can not be retained below the threshold value using RPA scheme.

- An $\varepsilon$-decomposition technique is used to decompose the network into multiple control areas. The proposed ASDC strategy, thus, is implemented for PV units located in the same control area.

The rest of the paper is organized as follows. First, Section 2 analyzes the problems of voltage rise and voltage sensitivity. Next, Section 3 presents the proposed control strategy followed by Section 4 describing the related modelling and simulation. Then, results and discussion presenting the efficacy of the proposed control strategy are given in Section 5. Finally, concluding remarks are drawn in Section 6.

\section{Problem analysis}

\subsection{Voltage rise issues}

Due to the increasing level of PV integration in LV distribution networks, voltage rise has become a more frequent issue. Voltage difference $(\Delta V)$ between the sending and receiving end voltage of a typical LV distribution line section with an impedance of $(R+j X)$ can be expressed as,

$\Delta V=\left(\frac{P-j Q}{V_{R}^{*}}\right)(R+j X)$

where $V_{R}^{*}$ denotes the conjugate of the receiving end voltage, $P$ and $Q$ denote the active and reactive power transferred through the line, respectively.

Further consider the receiving end bus supplies local power consumption of $P_{L}$ and $Q_{L}$ of loads, and receives local power generation of $P_{G}$ and $Q_{G}$ from PV systems. By ignoring the imaginary part, Eq. (1) can be rewritten as,

$|\Delta V| \approx \frac{\left(\left|P_{L}\right|-\left|P_{G}\right|\right) R+\left(\left|Q_{L}\right|-\left|Q_{G}\right|\right) X}{\left|V_{R}^{*}\right|}$

where $P=\left|P_{L}\right|-\left|P_{G}\right|$ and $Q=\left|Q_{L}\right|-\left|Q_{G}\right|$.

It can be inferred from Eq. (2) that when a high level of $P_{G}$ coincides with a low $P_{L},|\Delta V|$ is negative, indicating that voltage level rises towards the end of the line. In practice, if the voltage level measured at the POC exceeds 1.1 p.u., PV systems are disconnected from the network and are reconnected after some delay [12]. If the solar irradiance varies insignificantly, the PV systems continue switching off and on with the network. To solve the voltage rise issue, the reverse power flow needs to be limited by reducing $P_{G}$ and $Q_{G}$ from PV systems.

An important notice from Eq. (2) is that for a typical LV network with high $R / X$ ratio, changes in active power strongly impact the voltage magnitude variation. However, the reactive power control of PV inverters is, therefore, still beneficial for voltage control. If the PV inverters can operate with 0.9 power factor (PF), it can absorb reactive power with an amount of approximately $50 \%$ of the rated active power for grid ancillary services [27], which reduces the curtailment of active power injection. Some of commercial PV inverters are capable of providing such reactive power absorption as discussed in [28]. In this paper, the reactive power control strategy is further studied to mitigate voltage rise problems in LV distribution networks.

\subsection{Variation in impact of $P V$ generation on voltage regulation}

The variation in the impact of active and reactive power injection 
from PV inverters on phasor bus voltages and voltage angle varies depending on the bus location, which can be observed by applying the concept of voltage sensitivity matrix. The voltage sensitivity matrix is derived by reversing the system Jacobian matrix, which is resulted from solving the nonlinear load flow using Newton-Raphson algorithm [29].

Owing to the radial feature, the critical voltage rise in the LV networks due to the surplus PV power generation predominantly occurs at the bus located at the end of the feeder. According to [26], in a radial LV feeder the voltage sensitivity at the critical bus to active power and reactive power injection from PV systems both increase as the distance to the transformer increases along the feeder. Then, controlling active power and reactive power injection from PV systems connected to the buses with the higher impact sensitivity becomes more effective on voltage rise mitigation. It is, therefore, necessary to group PV systems with strong coupling nature, e.g., voltage sensitivity, for solving the voltage violation problems.

\section{Proposed control strategy}

The proposed control strategy aims to mitigate voltage rise along the feeder while significantly reducing the amount of curtailed PV generation. To this end, the $\varepsilon$-decomposition technique is utilized to decompose the network into multiple control areas containing groups of PV systems. The adaptive sequential droop control (ASDC) approach is then applied in the decomposed control areas to alleviate voltage rise issues.

Fig. 1 presents the block diagram of the proposed ASDC approach, which is embedded in each PV inverter, consisting of two layers: a local control and a coordination control layer. The former is based on a sequential droop control (SDC) mechanism, and the latter is based on a consensus-based distributed control (CBDC) mechanism. The local control layer is responsible for continuously monitoring and mitigating voltage rise conditions at the POC by regulating active and reactive power of PV units. The coordination control layer, which operates with a lower response speed than the local control layer, is responsible for adequately coordinating PV systems for the voltage level violations by periodically adapting the local control layer. As can be seen in Fig. 1, the output power set points of the PV inverters $\left(P^{\text {net }}, Q^{\text {net }}\right)$ are determined by the local control layer, which regulates the APC and RPA scheme of PV units in response to the voltage rise at the POC. Furthermore, the $P-V$ and $Q-V$ droop control parameters, i.e., the threshold levels $\left(V^{t P}\right.$ and $V^{a Q}$ ), are periodically set by the coordination control layer. The operation of this coordination control layer, therefore, can be regarded as a correction process to the local control layer in order to obtain the fair generation curtailment. To complete this process, the coordination control layer first exchanges the PV active power injection ratios $(\beta)$ between neighboring PV inverters, then reaches the common agreement of $\beta$ in an iterative manner. The proposed control strategy is discussed next in more detail.

\section{1. $\varepsilon$-Decomposition technique}

In order to determine various control areas for the ASDC approach, the decomposition of the network is done such that, the decomposed network consists of a series of sub-networks. Consider the fact that the voltage rise is a local problem, grouping these nodes into sub-networks, monitoring and solving the sub-networks independently appear promising [30]. Each sub-network bounds the coverage range of the communication network in this area. Given this boundary, the coordination mechanism can be executed among PV systems located in the same control area.

The $\varepsilon$-decomposition technique is identified by the graph theory. In this technique, a value of $\varepsilon$ is pre-defined with $0<\varepsilon<1$, which is regarded as a threshold of the interconnection strength among subareas. Let us assume a graph $\mathcal{G}=(\mathcal{V}, \xi)$ with the set of vertices $\mathcal{V}$ (i.e., $\mathrm{PV}$ units) and the set of edges $\xi$ (i.e., communication links between PV units). If there exists an edge between neighboring sub-graphs that is smaller than the threshold value of $\varepsilon$, such edge is specified as a weak connection of the graph [30]. This link, accordingly, will be disconnected from the graph and a series of sub-graphs will be identified. By mapping this decomposed graph to the original network, the decomposition of a network is attained with mutual coupling among sub-network not greater than a given $\varepsilon$ value [31]. A different series of decomposed sub-systems can be generated by varying $\varepsilon$ value as it decides the sub-system size [30].

More specifically, our study involves using $n \times n$ admittance matrix, $\mathbf{Y}$ for the implementation of the $\varepsilon$-decomposition method. The basic principle is to associate a graph $\mathcal{G}$ with the matrix $\mathbf{Y}$, identify the edges $\left(\mathcal{V}_{i}, \mathcal{V}_{j}\right) \subset \xi$ with magnitude smaller than or equal to a prescribed positive value of $\varepsilon$. Decomposed matrix $\overline{\mathbf{Y}}$ of original matrix $\mathbf{Y}$ is achievable if a permutation matrix $\mathbf{P}$ exists such that Eq. (3) is satisfied.

$\overline{\mathbf{Y}}=\mathbf{P}^{T} \mathbf{Y} \mathbf{P}$

where $\overline{\mathbf{Y}}$ is assumed as an $M \times M$ block matrix with $M_{p} \times M_{q}$ blocks $\overline{\mathbf{Y}}_{p q}=\left(\bar{y}_{i j}^{p q}\right)$ given by Eq. (4). P can be identified equivalently to dividing the graph $\mathcal{G}$ associated with $\mathbf{Y}$ into $M$ sub-graphs in the manner that the coupling magnitude of these sub-graphs in $\mathcal{G}$ less than $\varepsilon$ [30].

$\left|\bar{y}_{i j}^{p q}\right| \leq \varepsilon \quad \forall p, q \in N, p \neq q, i \in N_{p}, j \in N_{q}$

$\overline{\mathbf{Y}}$ can be conventionally represented as

$\overline{\mathbf{Y}}=\overline{\mathbf{Y}}_{0}+\varepsilon_{1} \overline{\mathbf{Y}}_{1}+\varepsilon_{2} \overline{\mathbf{Y}}_{2}+\cdots+\varepsilon_{m} \overline{\mathbf{Y}}_{m}$

where $\overline{\mathbf{Y}}_{0}$ is a block diagonal matrix and $\overline{\mathbf{Y}}_{1}, \cdots, \overline{\mathbf{Y}}_{m}$ are decomposed matrices with all elements less than or equal to $\varepsilon$, and $m$ is a series a prescribed positive value, $\varepsilon$ satisfying $\varepsilon_{1}>\varepsilon_{2}>\cdots>\varepsilon_{m}$. A comprehensive description of the $\varepsilon$-decomposition approach can be founded in [30].

\subsection{Local control layer: Sequential droop control}

In the local control layer, the SDC mechanism is implemented integrating $Q-V$ and $P-V$ droop control, as shown in Fig. $2(a)$. During normal operations, i.e., no voltage violations, the reactive power output of the PV inverter $i^{1}\left(Q_{i}^{\text {net }}\right)$ is set to zero, while the active power output $\left(P_{i}^{\text {net }}\right)$ is set to the maximum power point, $P_{i}^{M P P}$. If the voltage level at the POC $\left(V_{i}\right)$ exceeds the threshold level for reactive power absorption $\left(V_{i}^{a Q}\right)$, i.e., at point (1), RPA is activated according to the $Q-V$ droop control mechanism. As can be seen in Eq. (6), $Q_{i}^{\text {net }}$ is set following a linear function of $V_{i}$.

where $\bar{Q}_{i}$ is the maximum reactive power output and $V_{i}^{g Q}$ is the threshold level for reactive power injection.

If $V_{i}$ reaches the threshold level for active power curtailment $\left(V_{i}^{t P}\right)$ at point (2), $Q_{i}^{\text {net }}$ is set to $-\bar{Q}_{i}$, i.e., maximum amount, with the aim at reducing the active power curtailment for voltage rise mitigation. Concurrently, the PV inverter starts APC mode using $P-V$ droop control with $P_{i}^{\text {net }}$ being reduced following a linear function of $V_{i}$ as expressed in

\footnotetext{
${ }^{1}$ In this paper, it is assumed that the PV system $i$ is connected to the node $i$ of the distribution network.
} 
$Q_{i}^{\text {net }}=\left\{\begin{array}{l}\bar{Q}_{i} \frac{\left(V_{i}^{g Q}-V_{i}\right)}{\left(V_{i}^{g Q}-\underline{V}\right)}, \text { if } \underline{V} \leq V_{i}<V_{i}^{g Q} 0, \text { if } V_{i}^{g Q} \leq V_{i} \leq V_{i}^{a Q}-\bar{Q}_{i} \frac{\left(V_{i}-V_{i}^{a Q}\right)}{\left(V_{i}^{t P}-V_{i}^{a Q}\right)}, \text { if } V_{i}^{a Q}<V_{i} \leq V_{i}^{t P}-\bar{Q}_{i}, \text { if } V_{i}^{t P}<V_{i} \leq \bar{V} \\ \end{array}\right.$

$P_{i}^{\text {net }}= \begin{cases}P_{i}^{\mathrm{MPP}}, & \text { if } \underline{V}<V_{i} \leq V_{i}^{t P} P_{i}^{\mathrm{MPP}}-P_{i}^{\mathrm{MPP}} \frac{\left(V_{i}-V_{i}^{t P}\right)}{\left(\bar{V}-V_{i}^{t P}\right)}, \text { if } V_{i}^{t P}<V_{i}<\bar{V} 0, \text { if } V_{i} \geq \bar{V}\end{cases}$

Eq. (7).

where $[\underline{V} \bar{V}]=[0.91 .1]$, as specified in the European Standard EN 50160. Recognizing that this sequential droop control allows the PV inverters to operate in reactive power priority mode, implying that voltage regulation is first attempted through RPA. APC is triggered only if the voltage levels can not be maintained below the threshold value using RPA.

It is worth mentioning that solar irradiance and PV module temperature are constantly changing in time, then the maximum power is also varying. Thus, to function $P-V$ droop control in PV inverter $i$, the inverter needs to be capable of effectively estimating $P_{i}^{M P P}$ while operating at a curtailed active power output. Several maximum power point estimation (MPPE) methods for PV active power control have been proposed in [12,32-34]. In fact, in [12], the PV inverter periodically switches to maximum power point tracking (MPPT) mode performed by the inside MPPT algorithm for a certain time window, then recapturing the actual maximum power. However, the droop control function remains regularly deactivated and activated, making the power outputs largely fluctuated and then voltage rise problems not fully solved. An interesting alternative introduced in [32] utilizes irradiance and temperature measurements to extrapolate the datasheet information to actual PV module parameters, then fits a linear regression model to determine the polynomial function coefficients for MPPE. In [33,34], a least-squares curve fitting model is built based on a dataset of voltage and current measured at the dc side to realize MPPE. These two latter MPPE methods allow the PV inverters to continuously estimate the maximum power and simultaneously function the active power curtailment. Since the sequential droop control parameters will be periodically modified in a real-time manner by the consensus-based distributed control as described in the next section, the MPPE approaches in [32-34] appear to be promising ones. Nevertheless, none of these approaches is incorporated in this paper as it is assumed that the maximum power set-points are already provided by a MPPE method.

Note that, the maximum reactive power output $\bar{Q}_{i}$ in Eq. (6) varies with $P_{i}^{\text {net }}$ and it is calculated in compliance with the European Standard EN 50438, which stipulates that all distributed generation units connected to LV distribution networks must operate with PF ranging from

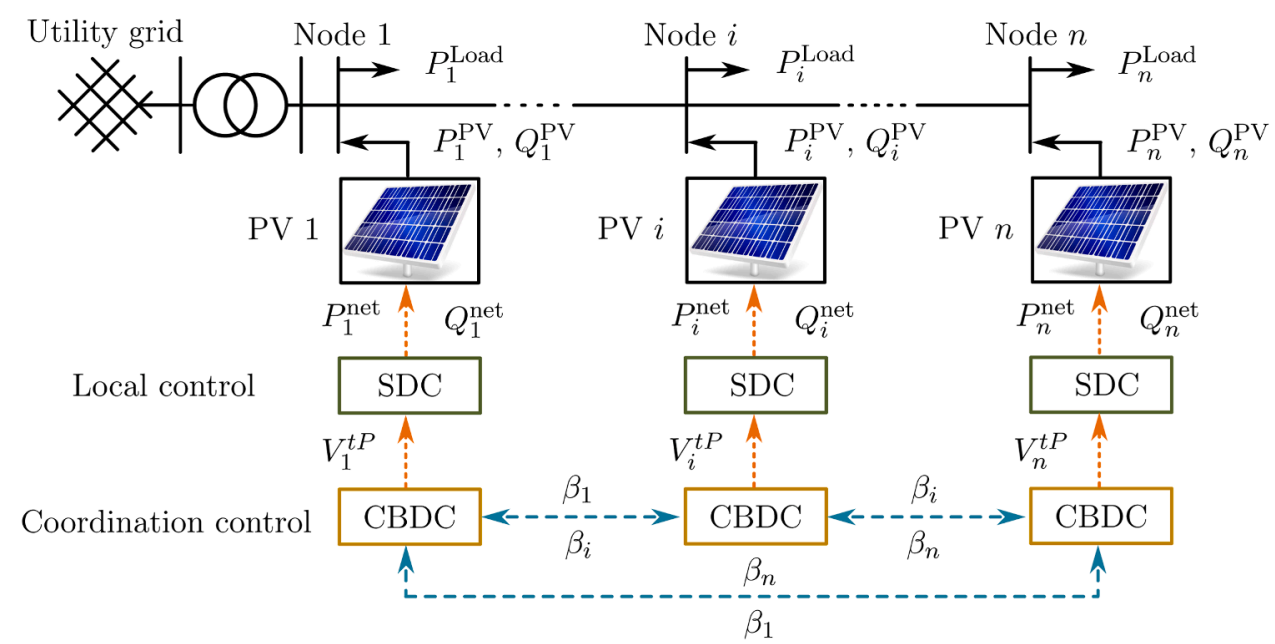

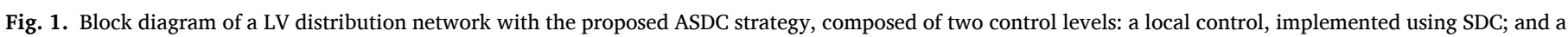

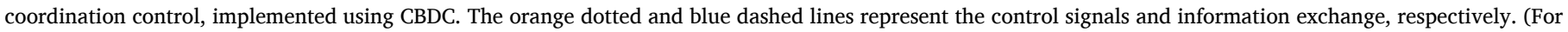
interpretation of the references to colour in this figure legend, the reader is referred to the web version of this article.) 
0.9 lagging to 0.9 leading [35]. Fig. 2 (b) shows the operation region of a $\mathrm{PV}$ inverter, which is within the shaded triangle, along with the apparent power $S$, rated active power $P_{i}^{\text {rated }}$, maximum reactive power $\bar{Q}_{i}$ specified by the PF requirement $(\cos \varphi=\mathrm{PF})$. The above-mentioned constraints of $\bar{Q}_{i}$ are integrated into the proposed control strategy as expressed below,

$\bar{Q}_{i 1}=\sqrt{\left(S_{i}\right)^{2}-\left(P_{i}^{\text {net }}\right)^{2}}$

$\bar{Q}_{i 2}=(\tan [\arccos (\mathrm{PF})]) P_{i}^{\text {net }}$

$\bar{Q}_{i}=\min \left(\bar{Q}_{i 1}, \bar{Q}_{i 2}\right)$

Nevertheless, applying the static $Q-V$ and $P-V$ droop control with the same threshold values in voltage rise conditions causes the deficient employment of reactive power capability and unequal generation curtailment of PV systems. In the radial LV feeders, PV units connected to the end of the feeder absorb significantly more reactive power and curtail more active power compared to other PV units. This can be regarded as an improper utilization of PV systems to the voltage regulation. Hence, a coordinated mechanism for enhancing the contribution of PV systems during voltage rise conditions need to be developed.

\subsection{Coordination control layer: Consensus-Based distributed control}

The improper utilization of PV inverter capability for voltage rise alleviation can be solved by the coordination control level, which is based on a CBDC mechanism. Consensus algorithms have been widely used as a basis for distributed control. Following this, each PV inverter communicates and shares its local information as the variable of interest with neighbours following a distributed procedure [36]. The goal of the consensus algorithm is to converge all PV inverters to a common agreement following an iterative manner. The variable of interest can be regarded as a quantity that is agreed by all PV inverters. The consensus algorithm considers a graph $\mathcal{G}=(\mathcal{V}, \xi)$ which represents the communication topology of the PV units. The set of nodes $\mathcal{V}=\{1, \ldots, n\}$ describes the set of PV inverters, whereas the set of edges $\xi \subset \mathcal{V} \times \mathcal{V}$ describes the set of communication links between PV inverters. Define for the PV inverter $i$ a variable of interest $x_{i}$ and define $\mathcal{N}_{i}=\{j \in \mathcal{V}:(i, j) \in \xi\}$ as the set of adjacent PV inverters that communicate with it. Considering the discrete nature of information exchange, the first-order consensus algorithm can be adopted. The discovery law of a variable of interest $x_{i}$ is then expressed as [36]

$x_{i}[k+1]=x_{i}[k]+\sum_{j \in \mathcal{N}_{i}} d_{i j}\left(x_{j}[k]-x_{i}[k]\right) \quad \forall i \in \mathcal{V}$

where $k$ is the iteration counter and $d_{i j}$ is the information weight factor between the unit $i$ and the neighbor unit $j$.

From a system point of view, the discovery law in Eq. (11) can be expressed in a matrix-vector form as

$\mathbf{X}[k+1]=\mathbf{D X}[k]$

where $\mathbf{X}[k]=\left[x_{1}[k], \ldots, x_{i}[k], \ldots, x_{n}[k]\right]^{T}$ and $\mathbf{X}[k+1]$ are the vectors of the discovered variables of interest at $k$ and $k+1$ iterations, respectively, and $\mathbf{D}$ is an information weighted matrix. As discussed in [37], if the values of D's elements are non-negative and the sums of D's rows and columns are all ones, the convergence of all PV inverters to a consensus will be reached at the average value of the variables of interest which can be expressed as

$\lim _{k \rightarrow \infty} \mathbf{X}[k]=\lim _{k \rightarrow \infty} \mathbf{D}^{k} \mathbf{X}[0]=\frac{\mathbf{I} \cdot \mathbf{I}^{T}}{n} \mathbf{X}[0]$

where $\mathbf{X}[0]$ is the initial value of $\mathbf{X}$ and $\mathbf{I}=[1,1, \ldots, 1]^{T}$. Notice from Eq. (13) that the convergence speed of the variable discovery procedure is a function of $\mathbf{D}$ [36]. Among various methods to define $d_{i j}$, the Metropolis method introduced in [38] is adopted in this paper, given by

$d_{i j}=\left\{\begin{array}{cl}1 /\left[\max \left(n_{i}, n_{j}\right)\right], & \text { if } i \neq j \forall j \in \mathcal{N}_{i} \\ 1-\sum_{j \in \mathcal{N}_{i}}\left(1 /\left[\max \left(n_{i}, n_{j}\right)\right]\right), & \text { if } i=j \\ 0 . & \text { Otherwise }\end{array}\right.$

where $n_{i}$ and $n_{j}$ represent the number of PV inverters that communicate with the PV inverter $i$ and $j$, respectively. To enable the consensus algorithm to properly operate, a sparse communication network is then established. In this communication network, each PV unit exchanges its local measured data only to adjacent nodes. Hence, neither a central controller nor the extensive high-bandwidth communication network is required.

In the proposed CBDC mechanism, the PV active power injection ratio $(\beta)$ is defined as the variable of interest, i.e., $x$ in Eq. (11). Following this, $\beta$ of each PV system is shared with the adjacent ones, given by

$\beta_{i}[k]=P_{i}^{\text {net }}[k] / P_{i}^{\mathrm{MPP}}[k] \quad \forall i \in \mathcal{V}$

where $P_{i}^{M P P}$ depicts the maximum power of the PV inverter determined by the local control as explained in Section 3.2.

Replacing $x_{i}$ in Eq. (11) by $\beta_{i}$, all PV units will converge to the same power injection ratio illustrated by

$\beta_{1}=\beta_{2}=\cdots=\beta_{n}$

As the consensus can only be reached when $k \rightarrow \infty$, this calls for an introduction of a stopping criterion by comparing the error at $k+1$ (a) SDC mechanism

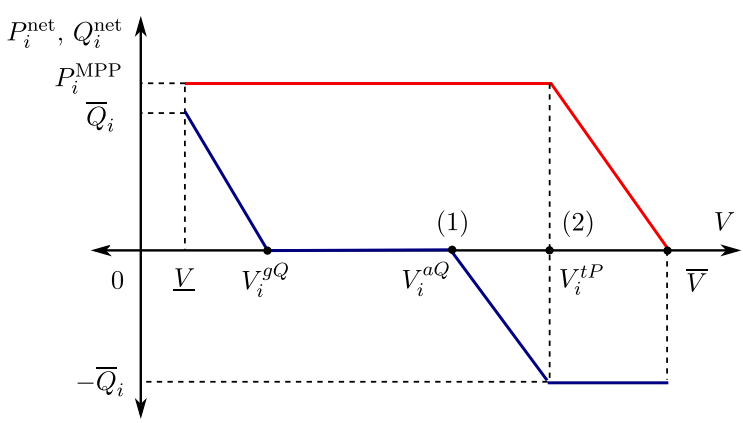

(b) PV inverter operation region

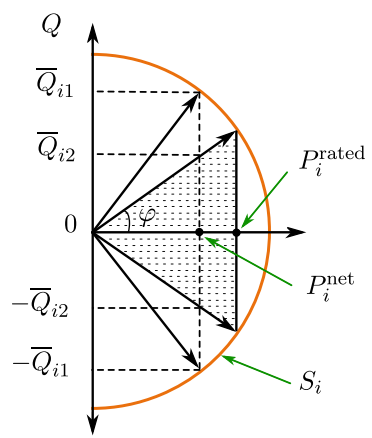

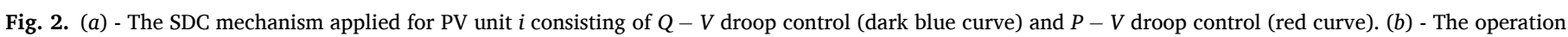

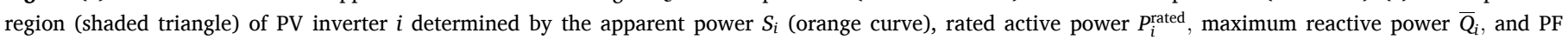
requirement $(\cos \varphi=\mathrm{PF})$. (For interpretation of the references to colour in this figure legend, the reader is referred to the web version of this article.) 


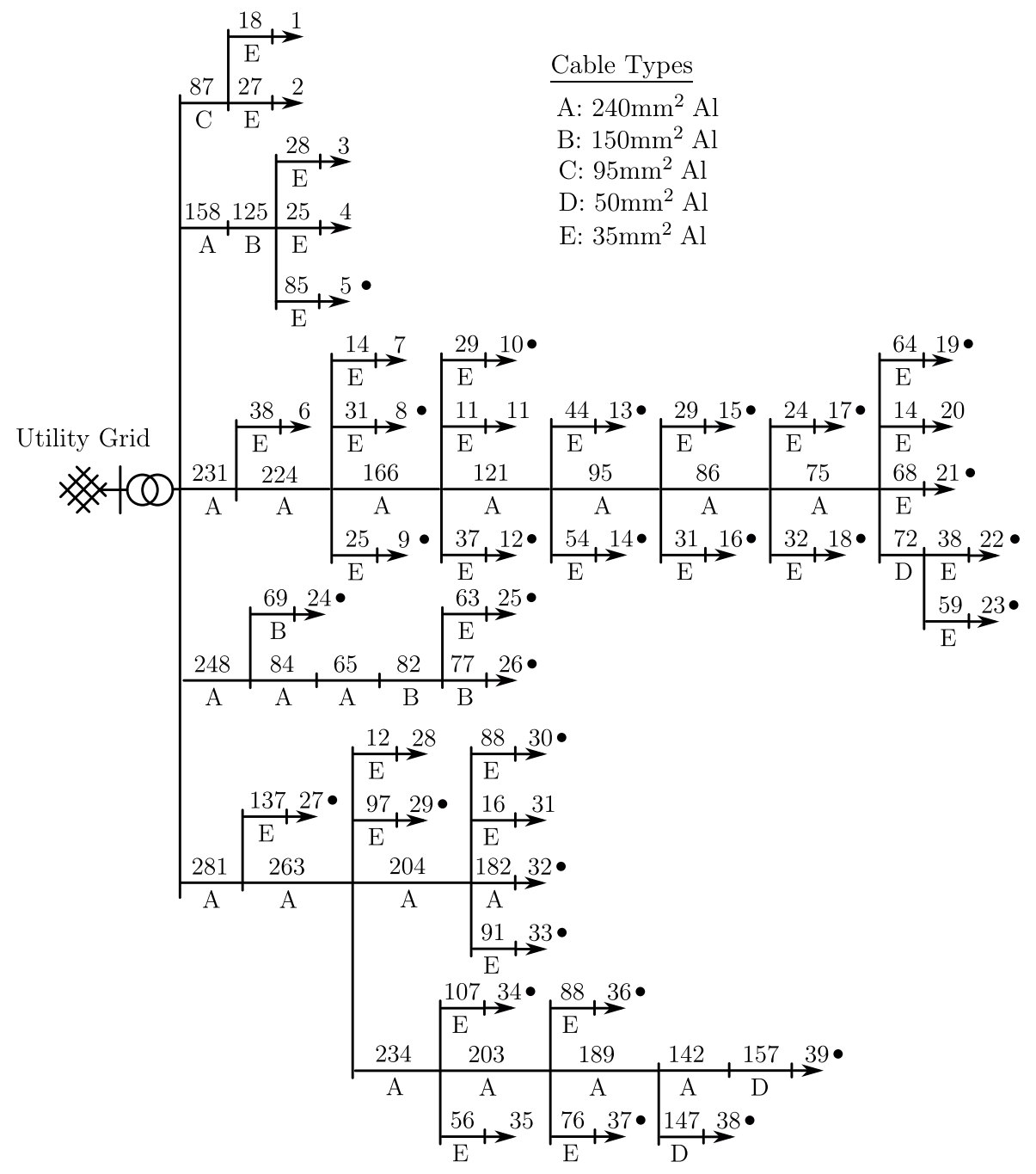

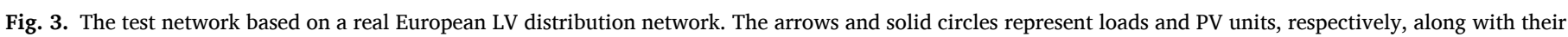
index. The numerical values along the power cable sections denote the cable length in meters, while the uppercase letters denote the cable types.

Table 1

$R, X$ values for the power cables of the test network.

\begin{tabular}{llll}
\hline Cable types & $R(\Omega / \mathrm{km})$ & $X(\Omega / \mathrm{km})$ & $R / X$ \\
\hline $240 \mathrm{~mm}^{2} \mathrm{Al}$ & 0.14 & 0.0818 & 1.71 \\
$150 \mathrm{~mm}^{2} \mathrm{Al}$ & 0.228 & 0.0844 & 2.70 \\
$95 \mathrm{~mm}^{2} \mathrm{Al}$ & 0.353 & 0.0868 & 4.07 \\
$50 \mathrm{~mm}^{2} \mathrm{Al}$ & 0.706 & 0.0924 & 7.64 \\
$35 \mathrm{~mm}^{2} \mathrm{Al}$ & 0.956 & 0.0982 & 9.74 \\
\hline
\end{tabular}

iteration with the consensus convergence tolerance $(\tau)$ as [36]

$\sum_{i \in \mathcal{V}}\left|\beta_{i}[k+1]-\beta_{i}[k]\right|<\tau \quad \forall i \in \mathcal{V}$

Having the updated value of $\beta_{i}$ at $k+1$ iteration, the coordination control level calculates the droop control parameter, i.e., $V_{i}^{t P}[k+1]$, and then updates the sequential droop control at the local control level with such new value. To complete this modification, the following rule is proposed.

$V_{i}^{t P}[k+1]=\frac{V_{i}[k+1]-\left(1-\beta_{i}[k+1]\right) \bar{V}}{\beta_{i}[k+1]}$

That adaptation of the droop control parameters, subsequently, enables the local control to command the PV inverters to fully employ the reactive power absorbing capability and properly share the curtailed active power if needed with other PV inverters. It is important to highlight that the coordinated mechanism is performed by PV inverters bounded on the coverage range of the corresponding communication topology. If the communication topology is built for a specific group of PV inverters, only the PV inverters within this group will follow the coordination. In this sense, the proposed ASDC can be implemented in various control areas in parallel.

\section{Modelling and simulation}

\subsection{Distribution network}

A real European LV distribution network is simulated in Matlab/ Simulink to investigate the effectiveness of the proposed approach. As depicted in Fig. 3, this network is energized from a $125 \mathrm{kVA}, 11 / 0.4 \mathrm{kV}$ distribution transformer and supplies electricity to 39 households, of which 28 are assumed to be equipped with PV units. The network comprises underground power cables with $R, X$ values being listed in Table 1 . The cable properties are provided in [39]. Since the proposed approach uses only changes in voltage magnitude as the trigger, the simulations were executed in the phasor mode. 


\subsection{Load profiles and PV system data}

The household loads in the test network are all uncontrollable and modelled using average normalized profiles of 400 residential customers, which are derived from smart meters with 15-minute resolutions [13]. The household load demands are in the ranges of $0.54-3.67$ $\mathrm{kW}$. The installed PV capacities are of residential-scale, ranging between 5.25 and $6.65 \mathrm{kWp}$. The total installed PV capacity is $168.3 \mathrm{kWp}$, depicting a penetration level of about $150 \%$, calculated as a ratio of total installed PV capacity to the maximum network load [40]. The input data for the PV systems, comprising the solar irradiation data and the ambient temperature data, are derived from real measurements. Due to the small geographical area of the test network, identical input data is used for all PV systems. For the compliance with the Standard EN 50438 [35], all PV inverters are assumed to operate within a PF of \pm 0.9 .

\subsection{Simulation setup}

The proposed control strategy is tested and compared with two other control methods as follows,

- Method 1: PV inverters are only embedded with the static SDC scheme (introduced in Section 3.2) with all the threshold values being constant over simulation time and uniform among PV inverters. Neither the coordination control nor the network decomposition is applied.

- Method 2: PV inverters employ the static $Q-V$ droop control and adaptive $P-V$ droop control. The threshold value for $Q-V$ droop control scheme remains constant over simulation time, while the threshold value for $P-V$ droop control scheme is updated by the coordination control layer (mentioned in Section 3.3). This method is adopted from our previous work presented in [24], where the network decomposition is not applied. This means that the coordination control is implemented for all PV inverters located in the same feeder, e.g., PV units from no. 8 to 23 will converge to a consensus of the power utilization ratio, while PV units from no. 27 to 39 will converge to the other consensus.

- Method 3: PV inverters are embedded with the proposed control strategy, i.e., ASDC, by which the threshold values for both $Q-V$ and $P-V$ droop control scheme are periodically modified. Reconsidering the aims of lowering the curtailed PV power generation during voltage rise conditions, this method is carried out along with the network decomposition.

In all these three methods, the local control of PV inverters employs

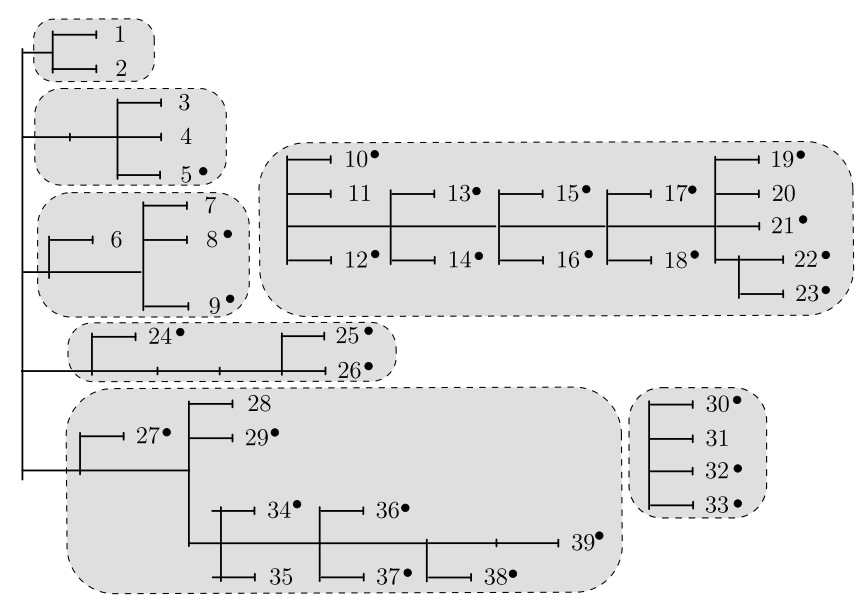

Fig. 4. Decomposition of the test network for $\varepsilon=0.21$ and determination of control areas as shaded regions. The solid circles represent PV units along with their index. the SDC scheme to tackle voltage rise issues. For the coordination control layer, a convergence tolerance $\tau=10^{-3}$ is applied as a stopping criterion for the consensus algorithm. Constant time duration of 15 seconds is used as the time interval at which the coordination mechanism updates the $P-V$ droop control parameter. The simulation is executed over two consecutive summer days.

\section{Numerical results}

\subsection{Network decomposition for control area determination}

Following the $\varepsilon$-decomposition technique described in Section 5.1, Fig. 4 depicts the determined 7 control areas for the test network with a chosen $\varepsilon$ value of 0.21 . With no loss of generality, this value of $\varepsilon$ is chosen to exemplify the decomposition procedure. To this end, $\mathbf{Y}$ matrix of the network is first computed and then normalized before partitioned with given $\varepsilon$ value into multiple decomposed matrices. The network was decomposed into sub-networks, in which each of them determines one of the control areas presented in Fig. 4. It is needed to be noted that, since the $\varepsilon$-decomposition technique bases on the admittance matrix $\mathbf{Y}$ of the network, recalculating the decomposition of the network is required when there is any change of the network configuration. In this paper, it is assumed that the test network configuration remains constant.

Once the network decomposition is completed, the corresponding sparse communication network, which is required for operating the coordination control layer, is established as shown in Fig. 5. As can be seen, the coverage range is within each determined control area, meaning that each PV unit only communicates with the other units located in the same area. The information weight factors $\left(d_{i j}\right)$ for the established sparse communication network are calculated using Eq. (14). Development of the communication infrastructure is considered more in the planning phase for the distribution network by the network operators, which involves long-term planning decisions. In this work, it is assumed that the communication infrastructure is already installed in the test distribution network and the main focus is on evaluating the proposed control strategy more in the operation phase. The planning requirement and cost for the communication infrastructure is, hence, considered beyond of the scope of this paper.

\subsection{Voltage rise mitigation}

Fig. 6 displays the resulting voltage magnitudes at the POCs of all PV systems in the network without any control mechanism over two consecutive summer days. This figure shows that voltage rise. e.g., above 1.07 p.u. only appears in the feeder no. 3 and 5 , where the PV units from no. 8 to 23 and the PV units from no. 27 to 33 are located, respectively. For the sake of comparison, the threshold value $V_{i}^{t P}=1.07$ p.u. is used uniformly for the static $P-V$ droop control in Method 1, and applied as a default setting for the adaptive $P-V$ droop control in Method 2 and 3. Meanwhile, the threshold value $V_{i}^{a Q}=1.06$ p.u. is applied uniformly for the static $Q-V$ droop control in both Method 1 and 2, and utilized as a default setting for the adaptive $Q-V$ droop control in Method 3. If no voltage rise is detected or if the PV units are not in operation (e.g., at night), Method 2 will automatically reset $V_{i}^{t P}$ to the default value, while Method 3 reset both $V_{i}^{a Q}$ and $V_{i}^{t P}$.

The decomposed test network along with the sparse communication network resulting from Section 5.1 are utilized to execute the simulation with Method 3. Recall that the simulation with Method 1 and 2 is performed without the network decomposition procedure. To evaluate the performance of the proposed control strategy, this study adopted an empirical cumulative distribution function (CDF) to visualize the maximum voltage levels occurring in the test network, as illustrated in Fig. 7. Without control, the voltage magnitude significantly rises (i.e., above 1.09 p.u.). Visibly, the curves of all three methods move far to the left, indicating that the network voltage represents considerably lower 

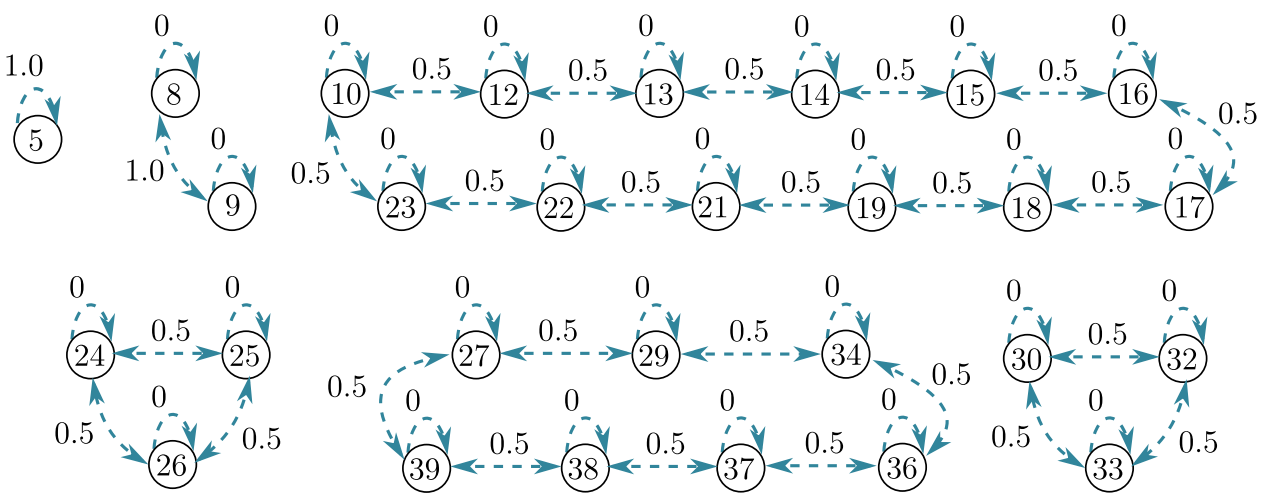

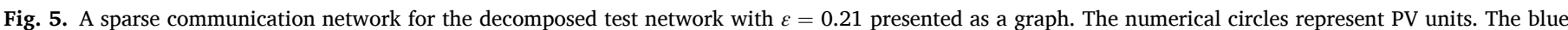

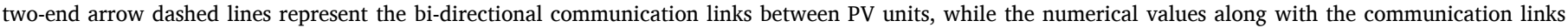

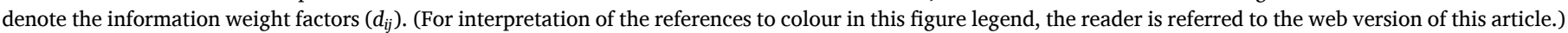

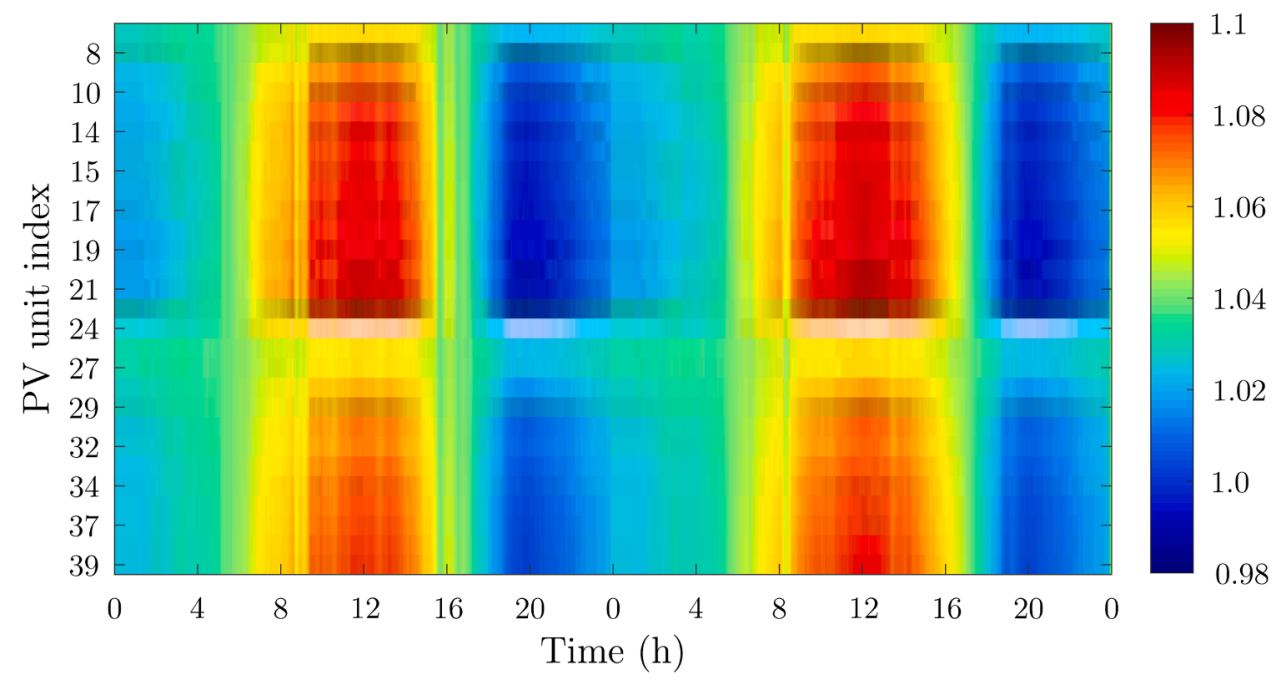

Fig. 6. Voltage magnitude at the POCs of PV systems in the network without any control mechanism.

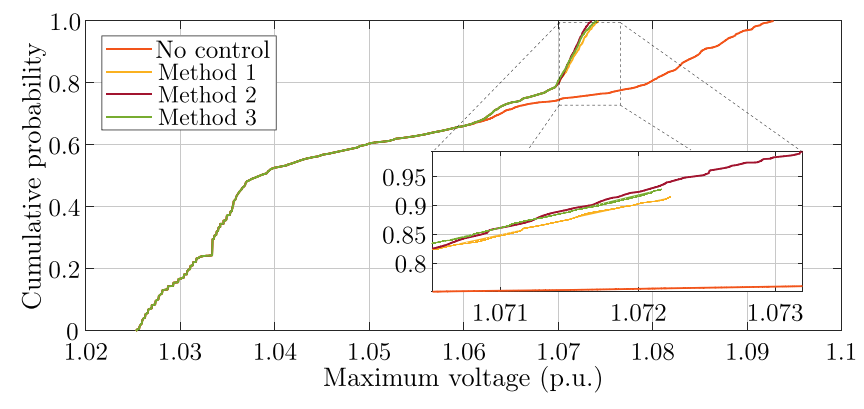

Fig. 7. Maximum voltage level measured in the network for different control methods using empirical cumulative distribution function (CDF).

Table 2

Summarized results for three control methods.

\begin{tabular}{llll}
\hline Properties & Method 1 & Method 2 & Method 3 \\
\hline $\begin{array}{l}\text { Maximum network voltage level (p.u.) } \\
\text { Total energy supplied from PV systems }\end{array}$ & 1.0743 & 1.0735 & 1.0740 \\
$\quad$ (kWh) & 2850.8 & 2833.1 & 2854.5 \\
$\begin{array}{l}\text { Total curtailed energy of PV systems (\%) } \\
\text { Total energy losses in the network (kWh) }\end{array}$ & 0.363 & 0.982 & 0.234 \\
\hline
\end{tabular}

magnitudes as compared to the case with no control. Additionally, it is observed that there is a slight difference in the CDF curves between each of the three control methods, meaning that the voltage profile of PV systems in three methods are comparable; therefore, all three methods effectively mitigate the voltage rise issues. This effective support is derived from employing the SDC scheme in the local controllers of the PV inverters, where both reactive and active power control are sequentially utilized.

Table 2 summarizes several performance metrics for three control methods. It is evident that Method 3 outperforms Method 1 and 2 in terms of the total curtailed PV energy. More specifically, Method 3 leads to a relatively similar value of the maximum voltage magnitude with the diminished percentage of curtailed energy (i.e., $0.234 \%$ ) compared to Method 1 and 2 (i.e., $0.363 \%$ and $0.982 \%$ respectively), corresponding with the reduction of $35.6 \%$ and $76.2 \%$ compared to Method 1 and 2, respectively. This superiority of Method 3 is derived from the increased reactive power absorption by PV systems compared with Method 1 and 2 , which is demonstrated in the next section. This growth in PV reactive power absorption along with a higher PV active power production (i.e., because of lower PV active power curtailment) causes Method 3 to have the slight rises of $1.63 \%$ and $3.88 \%$ in the network energy losses compared to Method 1 and 2, respectively. In three methods, Method 2 has the smallest value of energy losses, resulted from the lowest energy flow in the network. This is due to the smallest quantity of the energy supplied (kWh) of PV inverters in Method 2, meanwhile, its load energy consumption is in the same volume as Method 1 and 3. It can be noted 


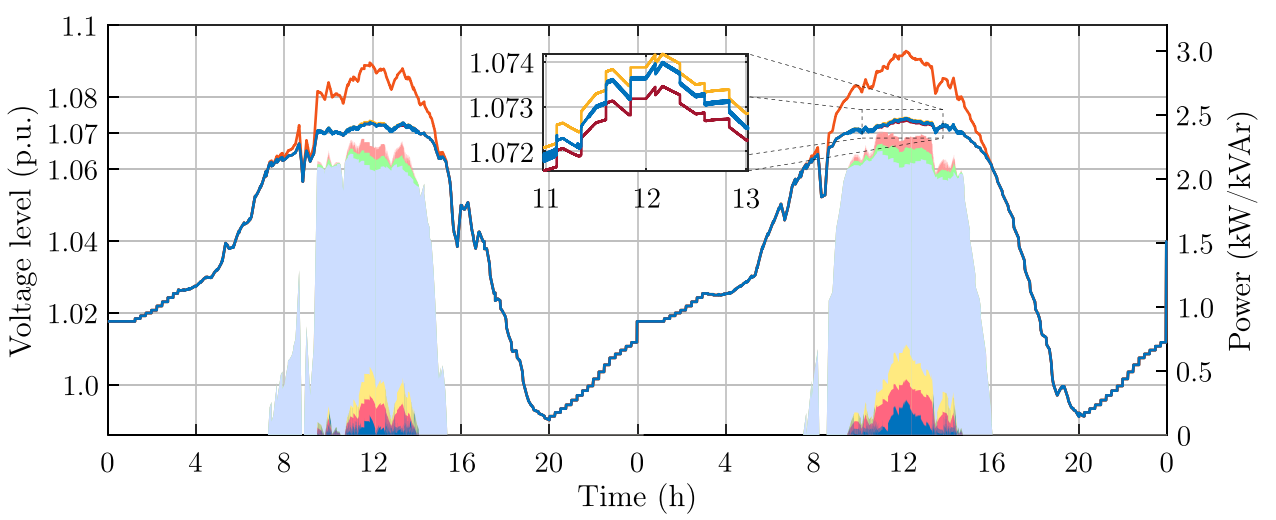

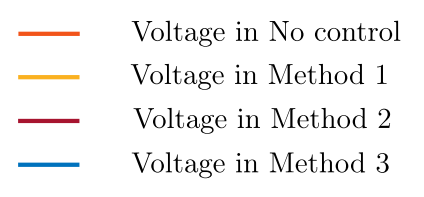

Absorbed $Q$ in Method 1

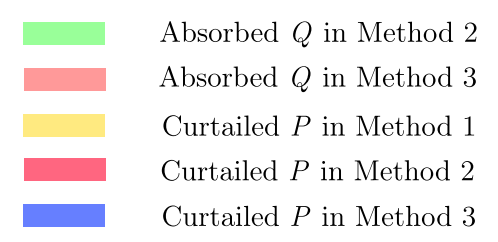

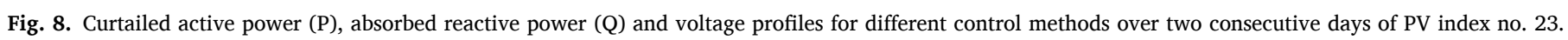

that the performance metrics analyzed in this section correspond with the predefined threshold values $V_{i}^{a Q}$ and $V_{i}^{t P}$. The sensitivity analysis of different threshold values is discussed in Section 5.4.

Another advanced feature of Method 3 is to significantly reduce the amount of curtailed active power of the PV units located at the end of the feeders (e.g., PV unit no. 23). To demonstrate this, Fig. 8 plots the calculated active power curtailment and reactive power absorption along with the measured voltage profiles of PV unit no. 23. At the POC of that PV system, the voltage rise above $V_{i}^{t P}=1.07$ p.u. predominantly occur (as displayed in Fig. 6). In cases of voltage rise, Method 1 results in the highest amount of curtailed active power compared to Method 2 and 3 (with a maximum value of approximately $0.53 \mathrm{~kW}$ and $0.71 \mathrm{~kW}$ in the first and second day, respectively). Owing to the static $Q-V$ droop control scheme and the consensus-based coordination control mechanism, Method 2 helps to prevent voltage rise situations with a smaller volume of active power reduction (with the maximum value being around $38.5 \%$ and $38.7 \%$ lower than Method 1 ). The total amount of curtailed energy of the PV system in Method 2 is $1.7343 \mathrm{kWh}$ (around $40.7 \%$ lower than that in Method 1). Finally, the proposed control strategy (i.e., ASDC) in Method 3 provides the comparable outcomes of voltage magnitude with the lowest amount of curtailed active power. In this method, the total curtailed PV energy is just nearly $0.69 \mathrm{kWh}$

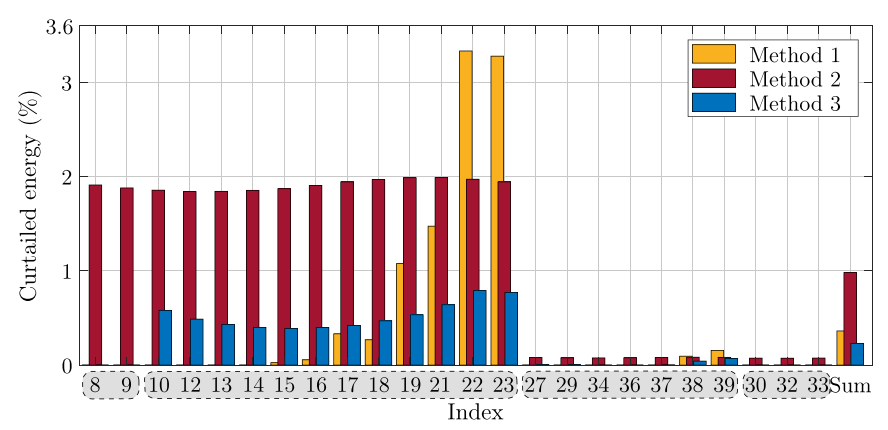

Fig. 9. Curtailed energy of the PV inverters for different control methods during voltage rise conditions as a percentage of individual PV energy (kWh) in the case with no control. Each index from 8 to 39 represents the corresponding individual PV unit, while the last index, i.e., Sum, represents the total amount. The shaded, rounded rectangles covering the PV index represent the groups of PV inverters within the corresponding control areas in Method 3 with value of $\varepsilon$ $=0.21$. (approximately 76.5\% and 60.3\% smaller than that in Method 1 and 2, respectively). Note from Fig. 8 that, the absorbed reactive power by the PV unit in Method 3 is higher than that in Method 2. This is because the curtailed active power in Method 3 is less than that in Method 2, leading to $P_{i}^{\text {net }}$ of PV inverters in Method 3 to become greater than Method 3. Accordingly, a greater $\bar{Q}_{i}$ is available for $Q-V$ droop control, as defined in Eq. (10). Hence, these findings show the effectiveness of the proposed control strategy to alleviate voltage rise problems as well as significantly decrease the volume of active power curtailment of the PV inverters.

\subsection{Contribution of various $P V$ systems to voltage rise mitigation}

To verify the contribution of the power control of various PV systems towards alleviating voltage rise issues, the curtailed energy of the PV inverters in the feeder no. 3 and 5 for different control methods as a percentage of the energy ( $\mathrm{kWh}$ ) in the case with no control are calculated, as presented in Fig. 9. Recall that voltage rise above the threshold setting, i.e., 1.07 p.u., only appears in the feeder no. 3 and 5, as discussed in Section 5.2. The reduction of active power required for voltage rise alleviation, as a result, is purely done on such feeders.

As can be seen, Fig. 9 illustrates that the effective contribution of PV generation curtailment to prevent voltage rise is obtained in Method 3 and also Method 2, in which the coordination control layer was applied. In Method 2, all PV inverters in the same feeder reduce their generation with a relatively equal percentage at the expense of a high volume of the total curtailed energy. In Method 3, although the share of energy reduction is not equal among PV inverters in the same decomposed

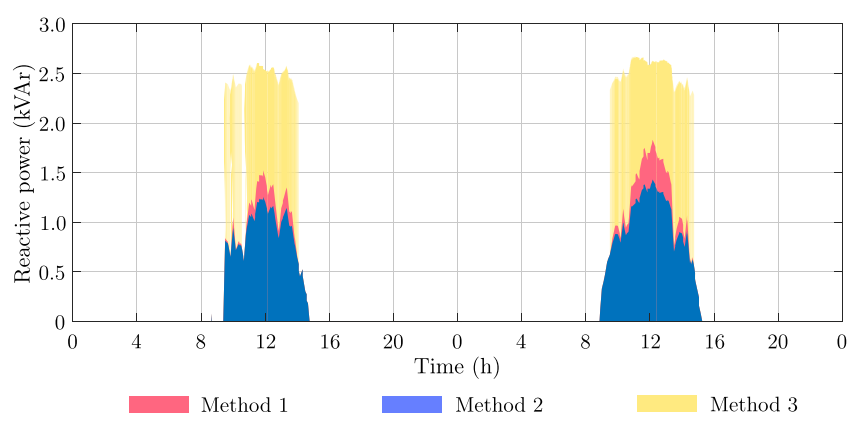

Fig. 10. Absorbed reactive power (Q) profiles for different control methods over two consecutive days of PV index no. 10. 
control areas (i.e., decomposition of the network with $\varepsilon=0.21$ as demonstrated in Fig. 4), the total curtail energy is remarkably reduced. The reason for the unequal reduction of curtailed energy in Method 3 is that the number of PV systems in several decomposed control areas is still large, e.g., $12 \mathrm{PV}$ systems in the control area for PV inverters from no. 10 to 23 . Then all PV inverters could not fully converge to a consensus of the power utilization ratio $(\beta)$. However, a smaller number of PV units enables the convergence to the consensus as shown in the following section. In contrast, being embedded only with the static SDC scheme, Method 1 caused large deviations of the curtailment among PV units. Noticeably, Method 3 has outstanding performance as compared to Method 1 and 2 because the percentages of total curtailed energy in Method 3 are substantially lower.

As depicted by the absorbed reactive power profile of PV unit no. 10 in Fig. 10, the efficient use of reactive power absorption is obtained in Method 3. Although PV unit no. 10 is located closer to the transformer, where the voltage rise conditions occur with lower levels (as can be observed in Fig. 6), reactive power absorption through the droop control is largely obtained. This is due to the use of the adaptive $Q-V$ droop control scheme, which is regulated by the coordination control mechanism for the control area of PV unit no. 10 as illustrated in Fig. 4. On the other hand, In Method 1 and 2, the location of PV unit no. 10 causes the static $Q-V$ droop control scheme to regulate reactive power absorption with considerably lower quantities compared to Method 3. Accordingly, the proposed coordination strategy proved to command PV inverters to not only more effectively contribute to voltage rise mitigation, but also lower their generation reduction.

Since voltage rise mitigation involves activating the local droop control schemes of multiple PV systems at the same time, fluctuation of PV power output can arise due to interaction between the local controllers. This power fluctuation can result in voltage oscillation in the network [2]. To damp the voltage oscillation, there are several techniques available in the literature, such as applying a low-pass filter represented by a continuous-time transfer function with a time constant for PV power output [2] and limiting the rate of changes of PV power outputs [41]. For this paper, the investigation of the voltage oscillation due to the interaction between PV droop controls is left for future researches.

\subsection{Sensitivity analysis of different threshold values}

As mentioned in Section 5.2, the threshold value settings for the local control play an important role in the performance of the control models. For further comparison of three control methods, the impact on the methods' performance metrics for different threshold values is analyzed in this section. The simulations are conducted using the configuration explained in Section 4 and Section 5.1 for two pairs of different threshold values for the droop control schemes. Key simulation results for three control methods are summarized in Table 3. It is observed in the first pair of threshold values $V_{i}^{a Q}=1.07$ p.u. and $V_{i}^{t P}=1.08$ p.u. that, Method 3 (i.e., the proposed control strategy) effectively solve the voltage rise issues and results in the decrease in the curtailed PV energy rate compared to Method 1 and 2, respectively. Regarding system energy losses, three control methods are insignificantly different. With the second pair of threshold values $V_{i}^{a Q}=1.08$ p.u. and $V_{i}^{t^{P}}=1.09$ p.u., the performance of three methods are equivalent. Since the voltage levels (i. e., 1.0873 p.u.) are all lower than $V_{i}^{t P}$ setting (i.e., 1.09 p.u.), PV active power curtailing mechanism in three methods remain inactivated.

As can be seen from Table 2 and Table 3, when voltage rise conditions activate the $P-V$ droop control scheme, Method 3, i.e., the proposed control strategy, induces the smallest quantity of $\mathrm{PV}$ active power reduction compared to Method 1 and 2, as well as adequately limits the voltage rise at the connection points. It is also important to highlight that, increasing threshold values for the droop control schemes allow the decrease in the active power curtailment, and power losses in the network as the differences between the network voltage levels and threshold values become smaller.

\subsection{Performance of the proposed control strategy for different control areas}

The performance of Method 3 is further evaluated using the network decomposition for various $\varepsilon$ values. The values of $\varepsilon$ were chosen to decompose the network into a different number of control areas. The percentages of curtailed energy of the PV inverters during voltage rise conditions in Method 3 with various $\varepsilon$ values are displayed in Fig. 11. The notable changes in the curtailed energy of individual PV units and also the total amount occur when different values of $\varepsilon$ are used. Nevertheless, the adequate contribution of PV power control towards voltage rise prevention is achieved as the percentages of curtailed energy are relatively comparable in each case of decomposition.

Notice also from Fig. 11 that, with $\varepsilon$ from 0.37 to 0.84 , the same curtailed energy rate is achieved for PV inverters within the same control areas. In case of $\varepsilon=0.44$, for instance, the control area for PV no. 19 and 21 has the curtailed energy percentage of $1.4 \%$, while in the control area for PV no. 22 and 23 the percentage is $3.3 \%$. This also illustrates that the introduction of the equal PV energy curtailment rate in each control area provokes the unequal PV energy curtailment rates between different control areas. The reason behind the fair reduction rates achieved with $\varepsilon$ from 0.37 to 0.84 is that the number of PV systems in the control areas is decreased, e.g., a maximum total of $4 \mathrm{PV}$ inverters within a control area for the case of $\varepsilon=0.37$ as compared to a maximum total of $12 \mathrm{PV}$ inverters for the case of $\varepsilon=0.21$. This smaller number of PV inverters allows the consensus of the power utilization ratio $(\beta)$ among them to be properly converged. To illustrate this, Fig. 12 shows the iteration number of the consensus of $\beta$ for a group of PV no. 22 and 23 in case of $\varepsilon=0.37$. The number of iteration is executed by the consensus algorithm to converge $\beta_{22}$ and $\beta_{23}$ of PV no. 22 and 23, respectively, to a common agreement, as considered by Eq. (17). For some duration, the iteration numbers reach high values due to the rapid changes in PV power generation as well as load consumption, which in turn induce the rapid changes in the voltage levels and then the power utilization ratio $(\beta)$ of PV systems. By contrast, the iteration number values are small when the voltage levels vary with low rate.

Furthermore, another number of performance metrics for Method 3 implemented with various $\varepsilon$ values are summarized in Table 4. This table points out that a larger $\varepsilon$ value leads to a growing number of control areas and a declining number of the bi-directional communication links between PV units as more weakly coupling terms between the network nodes are identified. With increasing values of $\varepsilon$ from 0.21 to 0.37 , the

Table 3

Comparison of different control methods with different threshold values.

\begin{tabular}{|c|c|c|c|c|c|c|}
\hline \multirow[t]{2}{*}{ Properties } & \multicolumn{3}{|c|}{$V_{i}^{a Q}=1.07$ p.u., $V_{i}^{t P}=1.08$ p.u. } & \multicolumn{3}{|c|}{$V_{i}^{a Q}=1.08$ p.u., $V_{i}^{t P}=1.09$ p.u. } \\
\hline & Method 1 & Method 2 & Method 3 & Method 1 & Method 2 & Method 3 \\
\hline Maximum network voltage level (p.u.) & 1.0809 & 1.0807 & 1.08 & 1.0873 & 1.0873 & 1.0873 \\
\hline Total energy supplied from PV systems (kWh) & 2860.8 & 2860 & 2861 & 2861.2 & 2861.2 & 2861.2 \\
\hline Total curtailed energy of PV systems (\%) & 0.014 & 0.042 & 0.007 & 0 & 0 & 0 \\
\hline Total energy losses in the network (kWh) & 63.39 & 63.33 & 63.46 & 61.12 & 61.12 & 61.12 \\
\hline
\end{tabular}




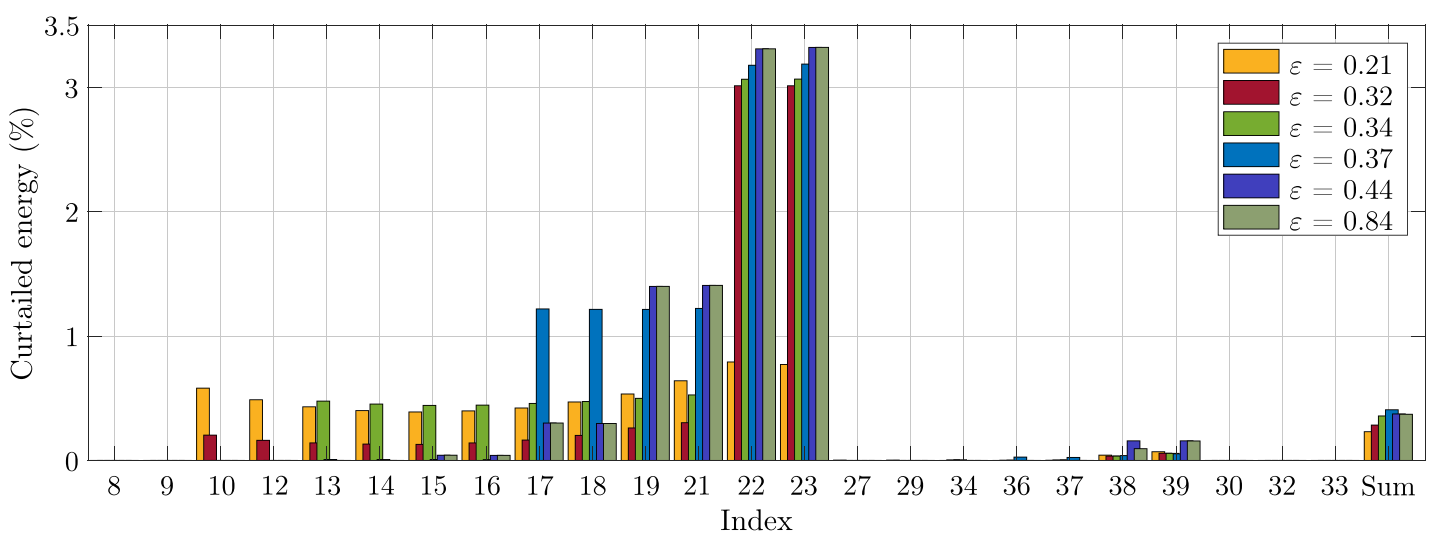

Fig. 11. Curtailed energy percentages of the PV inverters during voltage rise conditions in Method 3 with various $\varepsilon$ values. Each index from 8 to 39 represents the corresponding individual PV unit, while the last index, i.e., Sum, represents the total amount.

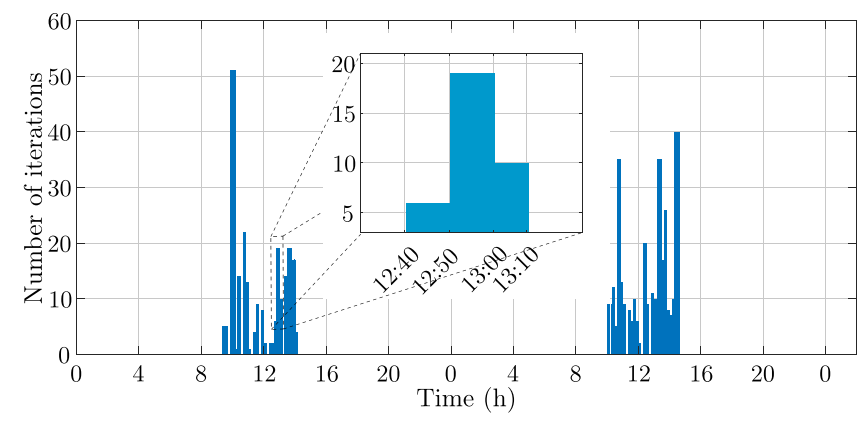

Fig. 12. Iteration number of the consensus algorithm applied in PV index no. 23 over two consecutive days. The zoomed-in figure in the middle shows the iteration number, i.e., the height of the bar represented in y-axis, for a time duration (hh:mm), i.e., the width of the bar represented in $\mathrm{x}$-axis.

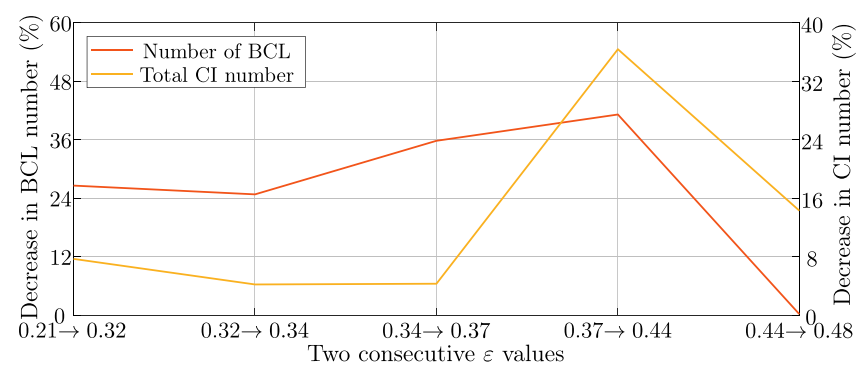

Fig. 13. Identifying $\varepsilon$ value. The $\mathrm{x}$-axis depicts two consecutive $\varepsilon$ values. The left y-axis represents the percentage decrease in the number of bi-directional communication links (BCL) associated with two successive $\varepsilon$ values, while the right $\mathrm{y}$-axis represents the percentage decrease in the consensus iteration (CI) numbers. percentage of total curtailed energy of PV systems increases. A reason for this is that the partitioned sub-networks, which each of them forms the control areas for PV systems, become gradually decreased in size. The coverage range of the proposed control strategy within individual control areas, subsequently, is for a small group of PV units. The decreasing number of PV units in the control area enables the iteration number for the consensus procedure to decrease, meaning that the consensus procedure converges with shorter time. The control areas, where voltage rise issues appear, receive less contribution from PV reactive power absorption to prevent voltage rise problems. Meanwhile, in the neighbouring control areas without voltage rise issues, the available PV reactive power absorption remains unused. Such decrease in the PV systems' active power generation and reactive power absorption provokes the decline of the energy losses in the network.

As also indicated from Table 4 that when $\varepsilon$ starts increasing from 0.44 to 0.84 , the resulting curtailed energy rates of PV inverters decline. Nevertheless, the variation of PV systems' curtailed energy is insignificant, causing the network energy losses to be remarkably similar. The rationale behind is that the size of the established control areas becomes small with a few PV units, e.g., a maximum total of $3 \mathrm{PV}$ inverters for the case of $\varepsilon=0.44$. The available $\mathrm{PV}$ reactive power absorption to support voltage rise mitigation, accordingly, is decreasingly exploited. Thus, the outcomes of adapting the SDC schemes of such PV inverters using the proposed control strategy (i.e., Method 3) are roughly similar to that of using the static SDC schemes, (i.e., Method 1), as shown in Table 2.

Recognizing that, when considering the number of bi-directional communication links (BCL) and total consensus iteration (CI) number, identifying $\varepsilon$ value can be based on the maximum percentage decrease in two successive values. Based on this evaluation criterion, $\varepsilon=0.44$ is the fitting value for the proposed control strategy applied in the given test network, as can be seen from Figure 13. This value of $\varepsilon=0.44$ also corresponds to a small amount of the total energy losses in the network, as illustrated in Table 4. However, when considering the total curtailed energy of PV systems (\%), the fitting value for $\varepsilon$ is the value corresponding to the lowest rate, that is $\varepsilon=0.21$. Hence, the long-term techno-economic analysis is required to select the optimal value for

Table 4

Summarized results of applying Method 3 with various $\varepsilon$ values.

\begin{tabular}{|c|c|c|c|c|c|c|}
\hline Properties & $\varepsilon=0.21$ & $\varepsilon=0.32$ & $\varepsilon=0.34$ & $\varepsilon=0.37$ & $\varepsilon=0.44$ & $\varepsilon=0.84$ \\
\hline Number of control areas & 7 & 9 & 10 & 12 & 16 & 22 \\
\hline Number of bi-directional communication links & 26 & 24 & 23 & 22 & 14 & 12 \\
\hline Total consensus iteration number & 2253 & 1653 & 1243 & 798 & 469 & 468 \\
\hline Maximum network voltage level (p.u.) & 1.0740 & 1.0739 & 1.0741 & 1.0741 & 1.0743 & 1.0743 \\
\hline Total energy supplied from PV systems (kWh) & 2854.50 & 2853.00 & 2850.90 & 2849.50 & 2850.46 & 2850.54 \\
\hline Total curtailed energy of PV systems (\%) & 0.234 & 0.287 & 0.360 & 0.409 & 0.375 & 0.373 \\
\hline Total energy losses in the network (kWh) & 69.13 & 68.60 & 68.26 & 67.95 & 67.99 & 68.00 \\
\hline
\end{tabular}


the epsilon decomposition. As the epsilon decomposition will define the size of the communication infrastructure and the curtailed PV energy rate, the long-term techno-economic involves balancing the investment in the communication infrastructure with the decrease in the losses of PV owners' revenue by reducing their curtailed power.

\section{Conclusion}

This paper presented an adaptive sequential droop control strategy for PV inverters to mitigate voltage rise problems in PV-rich LV distribution networks in a combination with the $\varepsilon$-decomposition technique. The $\varepsilon$-decomposition technique is employed to decouple the network into multiple control areas, where the proposed control strategy can be effectively implemented. The proposed control strategy includes the sequential $Q-V$ and $P-V$ droop control scheme used as the local control of PV inverters. A consensus-based distributed control mechanism is used to implement the coordination control among PV inverters, in charge of periodically adapting the parameters of both $Q-V$ and $P-V$ droop control scheme. The obtained results prove that the proposed control strategy adequately prevents voltage rise problems while significantly reducing the amount of total PV generation curtailment by approximately $35.6 \%$ and $76.2 \%$ when compared with the static sequential droop control and the static $Q-V$ droop control and adaptive $P-V$ droop control, respectively. Simultaneously, the effective contribution of all PV systems towards voltage rise mitigation is guaranteed. The performance of the proposed control strategy is further evaluated with multiple $\varepsilon$ values. Larger $\varepsilon$ values result in the fairness of PV generation curtailment, decline in system energy losses, and faster convergence for the consensus-based distributed control in the control areas, but in some cases increased volume of total curtailed energy of the PV systems. Selecting the optimal $\varepsilon$ value should be based on the long-term techno-economic analysis, which thoroughly assesses the investment in the communication infrastructure and the impact of reduced PV power curtailment on PV owners' revenue. Future research should involve investigating the potential impact of communication delays and failure on the consensus-based coordination control, as well as the voltage oscillation due to the interaction of multiple local droop controllers.

\section{CRediT authorship contribution statement}

Tam T. Mai: Conceptualization, Methodology, Software, Validation, Writing - original draft. Abu Niyam M.M. Haque: Resources, Writing review \& editing. Pedro P. Vergara: Writing - review \& editing. Phuong H. Nguyen: Resources, Writing - review \& editing, Project administration, Funding acquisition. Guus Pemen: Writing - review \& editing, Funding acquisition.

\section{Declaration of Competing Interest}

The authors declare that they have no known competing financial interests or personal relationships that could have appeared to influence the work reported in this paper.

\section{Acknowledgements}

This research have been funded by the ERA-Net Smart Grid Plus (m2M-GRID) project, http://m2m-grid.eu/.

\section{References}

[1] C. Long, L.F. Ochoa, Voltage control of PV-rich LV networks: OLTC-fitted transformer and capacitor banks, IEEE Trans. Power Syst 31 (5) (2016) 4016-4025, https://doi.org/10.1109/TPWRS.2015.2494627.

[2] P. Jahangiri, D.C. Aliprantis, Distributed volt / VAr control by PV inverters, IEEE Trans. Power Syst. 28 (3) (2013) 3429-3439, https://doi.org/10.1109/ TPWRS.2013.2256375.
[3] V. Klonari, J.F. Toubeau, T.L. Vandoorn, B. Meersman, Z. De Grève, J. Lobry, F. Vallée, Probabilistic framework for evaluating droop control of photovoltaic inverters, Electr. Power Syst. Res. 129 (2015) 1-9, https://doi.org/10.1016/j. epsr.2015.07.009.

[4] D.V. Bozalakov, J. Laveyne, J. Desmet, L. Vandevelde, Overvoltage and voltage unbalance mitigation in areas with high penetration of renewable energy resources by using the modified three-phase damping control strategy, Electr. Power Syst. Res. 168 (April 2018) (2019) 283-294, https://doi.org/10.1016/j. epsr.2018.12.001.

[5] P. Chaudhary, M. Rizwan, Voltage regulation mitigation techniques in distribution system with high PV penetration: a review, Renewable Sustainable Energy Rev. 82 (October 2017) (2018) 3279-3287, https://doi.org/10.1016/j.rser.2017.10.017.

[6] X. Su, M.A. Masoum, P.J. Wolfs, Optimal PV inverter reactive power control and real power curtailment to improve performance of unbalanced four-wire LV distribution networks, IEEE Trans. Sustain. Energy 5 (3) (2014) 967-977, https:// doi.org/10.1109/TSTE.2014.2313862.

[7] E. Dall'Anese, S.V. Dhople, B.B. Johnson, G.B. Giannakis, Optimal dispatch of photovoltaic inverters in residential distribution systems, IEEE Trans. Sustain. Energy 5 (2) (2014) 487-497, https://doi.org/10.1109/TEC.2014.2357997.

[8] T. Stetz, F. Marten, M. Braun, Improved low voltage grid-Integration of photovoltaic systems in germany, IEEE Trans. Sustain. Energy 4 (2) (2013) 534-542, https://doi.org/10.1109/TSTE.2012.2198925.

[9] M.J. Alam, K.M. Muttaqi, D. Sutanto, A multi-mode control strategy for VAr support by solar PV inverters in distribution networks, IEEE Trans. Power Syst. 30 (3) (2015) 1316-1326, https://doi.org/10.1109/TPWRS.2014.2344661.

[10] A. Samadi, E. Shayesteh, R. Eriksson, B. Rawn, L. Söder, Coordinated active powerDependent voltage regulation in distribution grids with PV systems, IEEE Trans. Power Del. 29 (3) (2014) 1454-1464.

[11] F. Olivier, P. Aristidou, D. Ernst, T.V. Cutsem, Active management of low-Voltage networks for mitigating overvoltages due to photovoltaic units, IEEE Trans. Smart Grid 7 (2) (2016) 926-936, https://doi.org/10.1109/TSG.2015.2410171.

[12] M.M. Viyathukattuva Mohamed Ali, M. Babar, P.H. Nguyen, J.F. Cobben, Overlaying control mechanism for solar PV inverters in the LV distribution network, Electr. Power Syst. Res. 145 (2017) 264-274, https://doi.org/10.1016/j. epsr.2016.12.002.

[13] A. Haque, P. Nguyen, T. Vo, F. Bliek, Agent-based unified approach for thermal and voltage constraint management in LV distribution network, Electr. Power Syst. Res. 143 (2017) 462-473, https://doi.org/10.1016/j.epsr.2016.11.007.

[14] R. Tonkoski, L.A.C. Lopes, T.H.M. El-fouly, Coordinated active power curtailment of grid connected PV inverters for overvoltage prevention, IEEE Trans. Sustain. Energy 2 (2) (2011) 139-147.

[15] L. Collins, J.K. Ward, Real and reactive power control of distributed PV inverters for overvoltage prevention and increased renewable generation hosting capacity, Renew. Energy 81 (2015) 464-471, https://doi.org/10.1016/j. renene.2015.03.012.

[16] T.T. Mai, A.N.M.M. Haque, T. Vo, P.H. Nguyen, Coordinated active and reactive power control for overvoltage mitigation in physical LV microgrids. 2018 International Conference on Renewable Power Generation, 2018, pp. 1-6.

[17] S.Y. Mousazade Mousavi, A. Jalilian, M. Savaghebi, J.M. Guerrero, Coordinated control of multifunctional inverters for voltage support and harmonic compensation in a grid-connected microgrid, Electr. Power Syst. Res. 155 (2018) 254-264, https://doi.org/10.1016/j.epsr.2017.10.016.

[18] P.P. Vergara, M. Salazar, T.T. Mai, P.H. Nguyen, H. Slootweg, A comprehensive assessment of PV inverters operating with droop control for overvoltage mitigation in LV distribution networks, Renew. Energy 159 (2020) 172-183, https://doi.org/ 10.1016/j.renene.2020.05.151.

[19] L. Steg, C. Vlek, Encouraging pro-environmental behaviour: an integrative review and research agenda, J. Environ. Psychol. 29 (3) (2009) 309-317, https://doi.org/ 10.1016/j.jenvp.2008.10.004.

[20] M.M.V.M. Ali, P.H. Nguyen, W.L. Kling, A.I. Chrysochos, T.A. Papadopoulos, G. K. Papagiannis, Fair power curtailment of distributed renewable energy sources to mitigate overvoltages in low-voltage networks. IEEE PES PowerTech 2015 Eindhoven, 2015, https://doi.org/10.1109/PTC.2015.7232796.Eindhoven

[21] S. Weckx, C. Gonzalez, J. Driesen, Combined central and local active and reactive power control of PV inverters, IEEE Trans. Sustain. Energy 5 (3) (2014) 776-784, https://doi.org/10.1109/TSTE.2014.2300934.

[22] S. Alyami, Y. Wang, C. Wang, J. Zhao, B. Zhao, Adaptive real power capping method for fair overvoltage regulation of distribution networks with high penetration of PV systems, IEEE Trans. Smart Grid 5 (6) (2014) 2729-2738, https://doi.org/10.1109/TSG.2014.2330345.

[23] M.A. Ghasemi, M. Parniani, Prevention of distribution network overvoltage by adaptive droop-based active and reactive power control of PV systems, Electr. Power Syst. Res. 133 (2016) 313-327, https://doi.org/10.1016/j. epsr.2015.12.030.

[24] T.T. Mai, A.N.M.M. Haque, P.H. Nguyen, Consensus-Based Distributed Control for Overvoltage Mitigation in LV Microgrids. IEEE PES Powertech 2019 Milano, 2019. Milan

[25] D. Shi, X. Chen, Z. Wang, X. Zhang, Z. Yu, X. Wang, D. Bian, A distributed cooperative control framework for synchronized reconnection of a multi-Bus microgrid, IEEE Trans. Smart Grid (2017) 1-10, https://doi.org/10.1109/ TSG.2017.2717806.

[26] E. Demirok, P.C. González, K.H. Frederiksen, D. Sera, P. Rodriguez, R. Teodorescu, Local reactive power control methods for overvoltage prevention of distributed solar inverters in low-voltage grids, IEEE J. Photovoltaics 1 (2) (2011) 174-182, https://doi.org/10.1109/JPHOTOV.2011.2174821. 
[27] G. Mokhtari, G. Nourbakhsh, F. Zare, A. Ghosh, Overvoltage prevention in LV smart grid using customer resources coordination, Energy Build. 61 (2013) 387-395, https://doi.org/10.1016/j.enbuild.2013.02.015.

[28] P.P. Vergara, T.T. Mai, A. Burstein, P.H. Nguyen, Feasibility and performance assessment of commercial PV inverters operating with droop control for providing voltage support services. 2019 IEEE PES Innovative Smart Grid Technologies Europe (ISGT-Europe), 2019, pp. 1-5, https://doi.org/10.1109/ ISGTEurope.2019.8905499.

[29] J.J. Grainger, W.D.J. Stevenson, Power system analysis, 1994, https://doi.org/ 10.1017/CBO9781107415324.004.

[30] D.D. Šiljak, Chapter 7 Nested Epsilon Decompositions. Decentralized Control of Complex Systems 184, 1991, pp. 374-413, https://doi.org/10.1016/S0076-5392 (08)63425-6.

[31] M.M.M. Ali, N.G. Paterakis, P.H. Nguyen, J.F. Cobben, A LV network overvoltage mitigation strategy based on epsilon-decomposition. 2017 IEEE PES PowerTech, IEEE, Manchester, England, 2017, pp. 1-6, https://doi.org/10.1109/ PTC.2017.7981197.

[32] A.F. Hoke, M. Shirazi, S. Chakraborty, E. Muljadi, D. Maksimovic, Rapid active power control of photovoltaic systems for grid frequency support, IEEE J. Emerg. Sel. Top. Power Electron. 5 (3) (2017) 1154-1163, https://doi.org/10.1109/ JESTPE.2017.2669299.

[33] E.I. Batzelis, G.E. Kampitsis, S.A. Papathanassiou, Power reserves control for PV systems with real-Time MPP estimation via curve fitting, IEEE Trans. Sustain. Energy 8 (3) (2017) 1269-1280, https://doi.org/10.1109/TSTE.2017.2674693.
[34] E.I. Batzelis, S.A. Papathanassiou, B.C. Pal, PV System control to provide active power reserves under partial shading conditions, IEEE IEEE Trans. Power Electron. 33 (11) (2018) 9163-9175, https://doi.org/10.1109/TPEL.2018.2823426.

[35] European Committee for Standards - Electrical, EN 50438:2013 - Requirements for micro-generating plants to be connected in parallel with public low-voltage distribution networks, 2013. 10.1109/TSTE.2014.2379918.

[36] M. Zeraati, M.E.H. Golshan, J.M. Guerrero, A consensus-Based cooperative control of PEV battery and PV active power curtailment for voltage regulation in distribution networks, IEEE Trans. Smart Grid 3053 (1) (2017) 1-11, https://doi. org/10.1109/TSG.2017.2749623.

[37] D. Li, B. Zhao, X. Zhang, L. Zhang, An improved droop control strategy for lowVoltage microgrids based on distributed secondary power optimization control, Energies 10 (1347) (2017), https://doi.org/10.3390/en10081080.

[38] A. Olshevsky, Linear Time Average Consensus on Fixed Graphs and Implications for Decentralized Optimization and Multi-Agent Control. 5th IFAC Workshop on Distributed Estimation and Control in Networked Systems, 2015, pp. 94-99, https://doi.org/10.1137/16M1076629.

[39] Nexans, Nexans Olex New Zealand Power Cable Catalogue, 2012, https://www.ne xans.co.nz/NewZealand/2013/PowerCableCatalogueFullversion2012.pdf. $10.1109 / 3477.931520$.

[40] T. Aziz, N. Ketjoy, PV Penetration limits in low voltage networks and voltage variations, IEEE Access 5 (2017) 16784-16792, https://doi.org/10.1109/ ACCESS.2017.2747086.

[41] P. Lusis, L.L.H. Andrew, A. Liebman, Interaction Between Coordinated and Droop Control PV Inverters. 11th ACM International Conference on Future Energy Systems, 2020, pp. 314-324, https://doi.org/10.1145/3396851.3397692. 